(c) American Dairy Science Association, 2006.

\title{
Fecal Shedding of Klebsiella pneumoniae by Dairy Cows
}

\author{
M. A. Munoz, C. Ahlström, B. J. Rauch, and R. N. Zadoks ${ }^{1}$ \\ Quality Milk Production Services, Cornell University, Ithaca, NY 14850-1263
}

\begin{abstract}
Klebsiella pneumoniae is a common cause of clinical mastitis in dairy cattle. Wood products are considered to be the main source of Klebsiella on dairy farms. Environmental hygiene and use of inorganic bedding materials such as sand are recommended to control Klebsiella mastitis. However, Klebsiella mastitis still occurs on well-managed dairy farms that use sand as bedding material. In a 5-mo study in a New York State dairy herd performed during the summer of 2005, all of 9 samples of unused sand bedding tested negative for Klebsiella, whereas 14 of 18 samples of used sand bedding contained Klebsiella at a median level of $10^{4.6} \mathrm{cfu} / \mathrm{g}$. We hypothesized that fecal shedding of Klebsiella by dairy cows contributes to the presence of Klebsiella in the environment. Using a cheap and simple method based on ampicillin-containing MacConkey agar for screening, and biochemical tests for confirmation of species identity, 595 fecal samples from healthy dairy cattle were screened for presence of Klebsiella. In a longitudinal study of 100 cows followed over 5 mo, more than $80 \%$ of fecal samples tested positive for $K$. pneumoniae. The average prevalence of $K$. pneumoniae-positive fecal samples was also above $80 \%$ in a cross-sectional study of 100 cows from 10 herds across New York and Massachusetts. Fecal shedding of $K$. pneumoniae by a large proportion of dairy cows may explain why Klebsiella mastitis occurs in herds that use inorganic bedding material or other bedding material that is free from Klebsiella upon introduction into the barn.
\end{abstract}

Key words: Klebsiella, mastitis, bedding, sand

\section{INTRODUCTION}

Mastitis is the most common and costly production disorder of dairy cattle and can manifest as subclinical infection or as clinical disease. In the United States, approximately $40 \%$ of clinical mastitis cases from which a pathogen is isolated are attributed to gram-negative organisms (Erskine et al., 1991). The most common

Received February 28, 2006.

Accepted March 31, 2006.

${ }^{1}$ Corresponding author: rz26@cornell.edu gram-negative causes of clinical mastitis are coliform bacteria, specifically Escherichia coli and Klebsiella spp. (Smith et al., 1985; Erskine et al., 1991; Todhunter et al., 1991). Klebsiella pneumoniae is by far the most common Klebsiella species infecting animals (Brisse and Duijkeren, 2005). It is also the most common Klebsiella species to cause mastitis (Erskine et al., 2002b; Roberson et al., 2004). Losses due to Klebsiella mastitis are much higher than losses due to $E$. coli mastitis in terms of milk production and survival (Erskine et al., 2002a; Gröhn et al., 2004). Klebsiella infections are less likely to respond to treatment and last significantly longer than $E$. coli infections (Smith et al., 1985; Roberson et al., 2004). The ratio of Klebsiella mastitis incidence to $E$. coli mastitis incidence varies from 1:10 to 1:1 across studies (Barkema et al., 1998; Roberson et al., 2004). In individual herds, especially those using J5 vaccination, the incidence and severity of Klebsiella mastitis can be higher than the incidence of $E$. coli mastitis (anecdotal information and culture data from herds participating in this study).

Considering the damage caused by infections, and the poor response of Klebsiella mastitis to treatment, prevention is of paramount importance in control of Klebsiella mastitis. The primary source of Klebsiella spp. in the cows' environment is organic bedding. Outbreaks of Klebsiella are commonly associated with sawdust bedding (National Mastitis Council, 1999). Klebsiella can be present in living wood (Bagley et al., 1978) and fresh sawdust bedding can be a source of Klebsiella mastitis (Newman and Kowalski, 1973; Hogan et al., 1989). Other organic bedding materials, such as recycled newspaper and straw also support growth of Klebsiella (Hogan et al., 1990). Inorganic materials such as crushed limestone and sand are least likely to harbor Klebsiella (Hogan et al., 1989). Rates of teat-end contamination and clinical mastitis have been correlated to bacterial loads in bedding (Hogan et al., 1989; Zdanowicz et al., 2004), and use of inorganic bedding is recommended as an effective means of environmental sanitation and mastitis prevention (National Mastitis Council, 1999). Klebsiella levels in clean or recycled sand are generally low, especially when the sand is first brought into the stalls (Kristula et al., 2005). During use, Klebsiella counts in bedding increase. Mean Klebsiella counts in sand bedding typically increased approximately 100 -fold in winter. In 
summer, up to 1,000-fold increases in Klebsiella counts could be observed (Kristula et al., 2005).

Clinical Klebsiella mastitis is more common in herds with low bulk milk SCC; that is, bulk milk SCC $<150,000$ cells $/ \mathrm{mL}$, than in herds with medium bulk milk SCC (between 150,000 and 250,000 cells/mL), indicating that it is of specific concern in well-managed dairy herds (Barkema et al., 1998). In New York State, many of the well-managed dairy herds that are serviced by Cornell University's Quality Milk Production Services use inorganic bedding material. Even so, complaints about the high incidence of clinical Klebsiella mastitis continue to exist in some herds. In humans, fecal shedding of Klebsiella plays an important role in pathogen dissemination (Podschun and Ullmann, 1998). We hypothesized that fecal shedding of Klebsiella spp. by dairy cows may contribute to the Klebsiella load in their environment. Intestinal carriage of $K$. pneumoniae in animals has been claimed to occur (Bagley and Seidler, 1977), but we were not able to locate any peer-reviewed studies documenting fecal shedding of Klebsiella in dairy cattle. The goal of our study, therefore, was to determine the prevalence of fecal shedding of Klebsiella by healthy, adult dairy cattle. A cross-sectional study was performed in the northeastern United States to determine the prevalence of fecal shedding in different herds, and a longitudinal study was performed to determine variability in the prevalence of fecal shedding in one herd over time. In the longitudinal study, Klebsiella counts of bedding from the stalls of the tested animals and from the unused sand pile were also determined. The testing methodology is cheap and simple and could easily be implemented in diagnostic laboratories.

\section{MATERIALS AND METHODS}

\section{Longitudinal Study}

A longitudinal study was carried out on a 1,200-cow dairy in central New York State from June through October 2005. Two pens in this herd, each holding approximately 200 lactating cows, were bedded with sand. Average incidence of clinical Klebsiella mastitis for these pens was 0.8 cases $/ 100$ cows per month. Over the duration of the project, 18 clinical Klebsiella mastitis cases occurred in the study pens. Twice a month, bedding samples were collected from the back one-third of $10 \%$ of stalls in both pens. In addition, a composite sample was taken from multiple points of the unused sand pile at approximately $25 \mathrm{~cm}$ depth. In each pen, 50 animals were selected for inclusion in longitudinal screening of fecal samples for presence of Klebsiella species. Cows were selected based on the expectation that they would stay in the same mid-to-late lactation cow pen for the next 5 mo. A fecal sample was collected from each cow once a month. Fecal

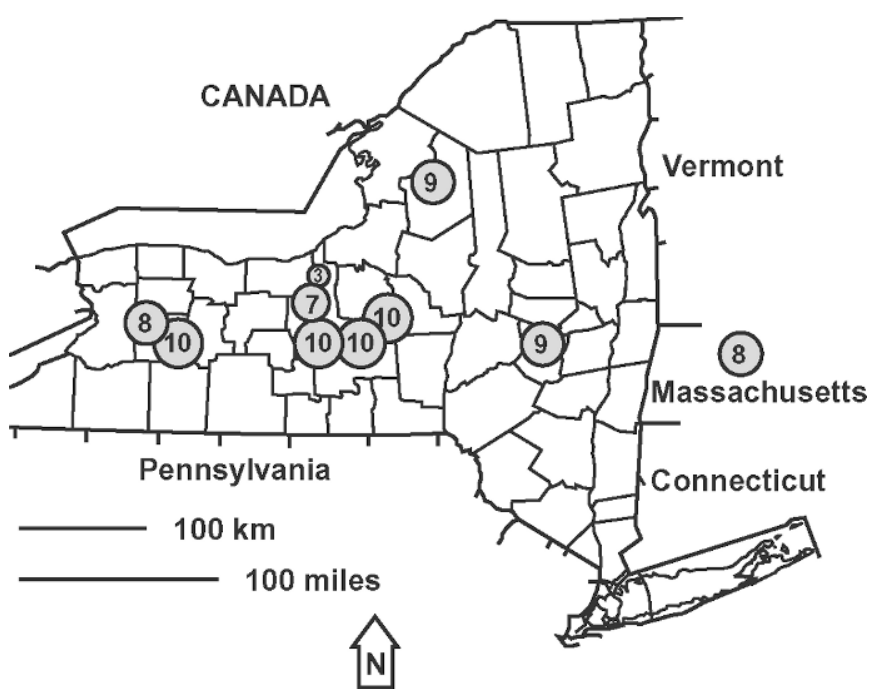

Figure 1. Location of herds participating in cross-sectional study on fecal shedding of Klebsiella in New York state. Per herd, 10 fecal samples were tested. Dot sizes are proportional to the number of positive samples, as shown; the smallest dot indicates 3 positive samples.

samples were collected from the rectum using individual palpation sleeves. To prevent cross-contamination, care was taken to avoid contact of the outside of the sleeve with anything but the sampled cow. Sleeves were turned inside out after sample collection and tied shut. Samples were transported to the laboratory in cooler boxes with ice packs.

\section{Cross-Sectional Study}

Fecal samples were collected from animals in 9 dairy herds throughout New York State and from one dairy herd in Massachusetts (Figure 1). Herd size ranged from 109 to 1,500 milking cows. In some herds, multiple animals had shown clinical Klebsiella mastitis in the past year. Klebsiella mastitis was not specifically reported as a problem in other herds. Within each herd, a convenience sample of 10 cows was chosen for fecal sample collection during routine health and fertility checks by herd veterinarians, or during herd surveys for mastitis conducted by Quality Milk Production Services personnel. Mastitis history of sampled cows was not taken into consideration. Sample collection and transportation were as described for the longitudinal study.

\section{Bedding Sample Processing}

Bedding ( $10 \mathrm{~g}$ ) was mixed with $90 \mathrm{~g}$ of sterile water and homogenized in a stomacher (MIX1, AES Laboratoire, Combourg, France) for $1 \mathrm{~min}$. Supernatant was decanted and used to prepare a 10 -fold serial dilution in sterile 
distilled water. Dilutions were plated with a Whitley Automatic Spiral Plater (Microbiology International, Frederick, MD) onto trypticase soy agar with 5\% sheep blood and $0.1 \%$ esculin (TSA-BE; PML Microbiologicals, Mississauga, ON, Canada), MacConkey agar, Edwards' medium, and Vogel Johnson medium (CM0115, CM0027, and CM 641, respectively, Oxoid Ltd., Basingstoke, UK) for determination of overall, coliform, streptococcal, and staphylococcal counts, respectively. Lactosepositive colonies surrounded by precipitate on MacConkey agar were considered to be $E$. coli and yellow or pink mucoid colonies without precipitate were considered to be Klebsiella. The lower limit of detection for coliform bacteria was $200 \mathrm{cfu} / \mathrm{g}$. Counts for used bedding were compared between pens using a 2 -sample $t$-test of logtransformed counts. Counts for used bedding were subsequently averaged and compared with zero using a 1sided, 1-sample $t$-test. Tests were performed with Statistix 8.0 (Analytical Software Co., La Jolla, CA), and significance was declared at $P<0.05$.

\section{Fecal Sample Processing}

Upon arrival in the laboratory, within $4 \mathrm{~h}$ of sample collection for the longitudinal study and within $24 \mathrm{~h}$ of sample collection for the cross-sectional study, the outside of each sleeve was wiped clean, and disinfected with $70 \%$ isopropyl alcohol. The tip of a finger was cut off the sleeve, and 7 to $10 \mathrm{~g}$ of fecal matter was transferred into an 11-mL snap-seal vial (Capitol Vial, Inc., Fultonville, $\mathrm{NY}$ ). Samples were stored at $4^{\circ} \mathrm{C}$ and processed further within $24 \mathrm{~h}$ of arrival for the longitudinal study or within $2 \mathrm{~d}$ of arrival for the cross-sectional study. A 1:10 dilution of fecal matter in saline was prepared by adding $1 \mathrm{~g}$ of fecal matter to $9 \mathrm{~mL}$ of saline in polystyrene culture tubes with dual-position polyethylene caps (VWR International, Batavia, IL). Samples were preincubated at $37^{\circ} \mathrm{C}$ for $4 \mathrm{~h}$ and streaked onto MacConkey containing ampicillin (MacA) using sterile cotton wool swabs, based on a modification of published methods (Degener et al., 1983): the original ampicillin concentration of $40 \mathrm{mg} / \mathrm{L}$ was reduced to $10 \mathrm{mg} / \mathrm{L}$, and only one dilution of the inoculum was plated. The lower ampicillin concentration allowed for growth of all Klebsiella isolates while inhibiting all $E$. coli isolates tested in a pilot experiment (data not shown). MacConkey agar was prepared following manufacturer's recommendations (MacConkey Agar No. 3, CM0115, Oxoid Ltd.). After autoclaving, media were tempered to $50^{\circ} \mathrm{C}$ and ampicillin (ampicillin sodium salt, Sigma-Aldrich Chemie GmbH, Steinheim, Germany) was added to a final concentration of $10 \mathrm{mg} / \mathrm{L}$. After the 4-h preincubation, fecal dilution vials were stored at $4^{\circ} \mathrm{C}$ in case they would be needed for further processing. The MacA plates were incubated overnight $(\sim 20 \mathrm{~h})$ at $37^{\circ} \mathrm{C}$.
For each set of samples, positive and negative controls were included. As positive control, a duplicate of one of the fecal samples was used and spiked with $1 \mu \mathrm{L}$ of fresh culture of Klebsiella pneumoniae ATCC 13883 in Todd Hewitt broth before preincubation. Saline was used as negative control.

\section{Preliminary Identification of Klebsiella spp.}

After overnight incubation, MacA plates were assessed visually for presence of Klebsiella-like colonies and other colonies. The following criteria were use to identify Klebsiella-like colonies on MacA: small to large (1 to $7 \mathrm{~mm}$ range), dome-shaped, wet, mucoid, glistening colonies with smooth edges, without precipitate in the surrounding agar. Colonies had a pink center and pink edges, or a yellow center with pink or yellow edges. For most samples, the density of growth on MacA allowed for good visual assessment of colony morphology when the sample had been preincubated for $4 \mathrm{~h}$ before streaking (standard preincubation). For samples yielding no growth or very heavy growth on MacA, prolonged preincubation or no incubation of the fecal sample was used, as described below.

For MacA plates that showed no growth of Klebsiellalike colonies and fewer than 200 colonies of other morphological appearance, the vial with 1:10 fecal:saline dilution was taken from the refrigerator and incubated at $37^{\circ} \mathrm{C}$ for an additional $20 \mathrm{~h}$ (prolonged preincubation). Colony counts were approximated by counting of colonies on 1 quadrant of the MacA plate using a handheld colony counter. Plates that contained between 200 and 800 colonies but no Klebsiella-like colonies were considered to be Klebsiella negative. When more than approximately 800 colonies were present on a plate, it was difficult to assess presence or absence of Klebsiella-like colonies, and plates were considered to be too crowded to evaluate. For fecal samples yielding overcrowded plates, a new 1:10 dilution of the original fecal sample in saline was prepared and swabbed onto MacA without preincubation. The MacA plates that were streaked after prolonged or no preincubation of the fecal samples were incubated and interpreted as described for the standard preincubation.

Isolated Klebsiella-like colonies identified on MacA were subcultured onto TSA-BE for further testing. Klebsiella-like colonies that were in contact with adjacent colonies on the primary MacA plate were subcultured onto a secondary MacA plate to obtain isolated colonies. Isolated colonies from the secondary MacA plate were then subcultured onto TSA-BE. Per fecal sample, up to 4 Klebsiella-like colonies were subcultured on TSA-BE and used for biochemical confirmation of species iden- 
tity. The cost of supplies for fecal sample testing (commercial pricing) was approximately $\$ 1.60$ per sample.

\section{Confirmation of Species Identity}

To confirm the identity of putative Klebsiella isolates, citrate and motility tests were performed as recommended by the National Mastitis Council (National Mastitis Council, 1999). Cultures on TSA-BE were visually checked for purity, and subsequently inoculated on Simmons citrate agar slants (Simmons citrate agar, Oxoid Ltd.) using sterile 1- $\mu \mathrm{L}$ loops, and stabbed into vials with motility-indole-ornithine medium (Difco, Becton, Dickinson and Company, Sparks, MD) using sterile culture needles. Citrate slants and motility-indole-ornithine vials were incubated for approximately $16 \mathrm{~h}$ at $37^{\circ} \mathrm{C}$. When results of the citrate test were inconclusive, incubation was continued for an additional $24 \mathrm{~h}$. The citrate test was considered positive when the green medium turned blue. Klebsiella pneumoniae ATCC 13883 was used as positive control and E. coli ATCC 25922 as negative control. For motility testing, cloudiness throughout the medium was considered a positive reaction. Escherichia coli ATCC 25922 was used as positive control and $K$. pneumoniae ATCC 13883 as negative control. The indole reaction was not read from the motility-indole-ornithine vial but was tested independently using the indole spot test (BBL DMACA indole reagent droppers, Becton, Dickinson and Co.). For the indole test, $E$. coli ATCC 25922 was used as positive control and $K$. pneumoniae ATCC 13883 as negative control. Isolates were considered to belong to the genus Klebsiella if they were citrate positive and motility negative. Indole-positive Klebsiella isolates were considered to belong to the species Klebsiella oxytoca and indole-negative isolates were considered to belong to the species Klebsiella pneumoniae. Cows were considered to be shedding $K$. pneumoniae or K. oxytoca if at least one Klebsiellalike colony from the fecal sample was confirmed to belong to the respective species. The cost of supplies for confirmation of species identity (commercial pricing) was approximately $\$ 1.96$ per isolate.

\section{RESULTS}

\section{Longitudinal Study}

All samples from the unused sand pile tested negative for Klebsiella, whereas 14 of 18 (78\%) of used bedding samples tested positive, with Klebsiella counts ranging from $8 \times 10^{3}$ to $8 \times 10^{5} \mathrm{cfu} / \mathrm{g}$. The median count was $10^{4.5}$ cfu/g for used bedding (Table 1). Klebsiella counts in used bedding did not differ between the 2 pens that were monitored longitudinally ( 2 -sample $t$-test of log-
Table 1. Proportion of Klebsiella-positive fecal samples, and Klebsiella counts in bedding samples during a longitudinal study in a New York State dairy herd in $2005^{1}$

\begin{tabular}{|c|c|c|c|}
\hline \multirow[b]{2}{*}{ Month } & \multirow[b]{2}{*}{ Fecal $(\%)$} & \multicolumn{2}{|c|}{ Sand (log cfu/g) } \\
\hline & & Unused & Used \\
\hline \multicolumn{4}{|l|}{ June } \\
\hline First half & 77 & $\mathrm{ND}^{2}$ & 4.4 \\
\hline Second half & $\mathrm{NT}^{2}$ & ND & ND \\
\hline \multicolumn{4}{|l|}{ July } \\
\hline First half & 77 & ND & 4.8 \\
\hline Second half & NT & ND & ND \\
\hline \multicolumn{4}{|l|}{ August } \\
\hline First half & 87 & ND & 5.8 \\
\hline Second half & NT & ND & 4.1 \\
\hline \multicolumn{4}{|l|}{ September } \\
\hline First half & 82 & ND & NT \\
\hline Second half & NT & ND & 4.6 \\
\hline \multicolumn{4}{|l|}{ October } \\
\hline First half & NT & ND & 4.7 \\
\hline Second half & 84 & ND & 4.6 \\
\hline Average/median ${ }^{3}$ & 81.4 & ND & 4.5 \\
\hline
\end{tabular}

${ }^{1}$ Per month, 100 cows were tested, with the exception of October $(\mathrm{n}=95)$.

${ }^{2} \mathrm{NT}=$ not tested; $\mathrm{ND}=$ not detected.

${ }^{3}$ Average for proportion of positive cows; median for log-transformed counts in bedding, including samples in which Klebsiella was not detected.

transformed counts, $P>0.9)$. Klebsiella counts in used bedding were significantly greater than zero $(P<0.0001)$.

In June, July, August, and September, 100 animals were tested, and 95 of those animals were available for testing in October. Prevalence of fecal shedding of $K$. pneumoniae was $77,77,87,82$, and $84 \%$ for each consecutive month, respectively, and $81.4 \%$ across the total of 495 fecal samples (Table 1). The majority of positive samples (381 of 403 or $81 \%$ of positive samples) were detected using the 4-h preincubation. Prolonged incubation was used for 71 of 495 samples (14\%). Klebsiella pneumoniae was detected in 22 of 71 samples after prolonged incubation; that is, in $31 \%$ of samples exposed to prolonged incubation, or $5.5 \%$ of all positive samples. Of 481 Klebsiella-like colonies obtained from fecal samples after standard or prolonged incubation, $93 \%$ were confirmed as $K$. pneumoniae. Fecal $K$. oxytoca was not detected in the longitudinal study. The majority of colonies that grew on MacA but did not have Klebsiella-like morphology were pink to red, flat colonies surrounded by a pink zone of precipitated bile salts. These colonies had a fecal odor. This morphology is typical for $E$. coli. Species identity of presumptive $E$. coli isolates from fecal samples was not confirmed through biochemical testing.

\section{Cross-Sectional Study}

Of 100 cows from 10 herds (10 per herd), 84\% tested positive for Klebsiella spp. in feces. The number of Kleb- 
siella-positive animals ranged from 3 to 10 of 10 tested (Figure 1). In 8 of 10 herds, 8 or more animals tested positive for fecal Klebsiella. Of 197 Klebsiella-like isolates that were identified based on colony morphology on MacA, 183 (93\%) were confirmed as Klebsiella spp. by means of biochemical testing. In one fecal sample, 2 colonies of K. oxytoca were identified. All other Klebsiella isolates were indole negative and were considered to be K. pneumoniae. Most positive samples ( 73 of 84 , or $87 \%$ of positive samples) were identified after 4-h incubation. Seven plates were initially Klebsiella-negative, and testing was repeated after prolonged incubation. After prolonged incubation, presence of $K$. pneumoniae was confirmed for 2 of 7 samples (29\%). Sixteen samples yielded plates that were too crowded to identify putative Klebsiella colonies and new plates were streaked from these samples without preincubation. Klebsiella was identified on 9 of 16 plates that were restreaked without preincubation. The 9 samples that were identified as K. pneumoniae-positive after restreaking without preincubation originated from a single herd, and had been transported to the laboratory without adequate cooling.

\section{DISCUSSION}

In a longitudinal study including almost 500 fecal samples collected from 100 healthy adult dairy cows in 1 herd over a 5-mo period, as well as in a cross-sectional survey of 100 fecal samples collected from 10 healthy adult dairy cows in each of 10 herds, prevalence of fecal shedding of $K$. pneumoniae was over $80 \%$. In the longitudinal study, Klebsiella was not detected in unused sand on any of 9 tests, whereas 14 of 18 used sand samples were Klebsiella positive.

Klebsiella mastitis is usually attributed to the presence of the bacteria in organic bedding material, particularly wood by-products (Hogan et al., 1989; Zdanowicz et al., 2004). However, Klebsiella can also be present in sand, with average numbers ranging from 100 to 2,200 $\mathrm{cfu} / \mathrm{g}$ for clean or recycled sand taken from the pile, to more than 100,000 cfu/g in sand taken from stalls during summer (Kristula et al., 2005). Klebsiella levels in sand reported by Kristula and coworkers (2005) are similar to the results from our study, which was conducted in summer: $<200 \mathrm{cfu} / \mathrm{g}$ for the pile and $10^{4.5} \mathrm{cfu} / \mathrm{g}$ (median value) for used sand. The large increase in Klebsiella numbers after introduction of sand into stalls implies that Klebsiella multiplies in sand, or that additional Klebsiella is contributed from other sources. Considering the high prevalence of fecal shedding of $K$. pneumoniae and the fact that there is direct contact or mixing of feces and bedding, feces are a very likely source of bedding contamination. The fact that cows harbor $K$. pneumoniae in their gastrointestinal tract implies that construction of new facilities, as considered by one of our clients to solve a Klebsiella mastitis problem, is no guarantee that Klebsiella will be eliminated from the herd environment. Fecal carriage of Klebsiella is also common in humans, with prevalence ranging from $5 \%$ in the general population to 77\% in hospital settings (Degener et al., 1983; Podschun and Ullmann, 1998). Early reviews of K. pneumoniae or Klebsiella spp. ecology mention gastrointestinal carriage by animals, including cattle, but do not provide data to support this claim, or quantification of the prevalence of fecal shedding (Bagley and Seidler, 1977; Bagley, 1985).

The focus of the current study was to determine the prevalence of fecal shedding of Klebsiella by dairy cattle rather than to correlate the prevalence of fecal shedding with bedding counts of Klebsiella, or to compare strains of Klebsiella between feces, bedding, and cases of clinical mastitis. Strain-level comparisons of fecal and environmental isolates with clinical mastitis isolates have been performed for other pathogens; that is, E. coli (Nemeth et al., 1994) and Streptococcus uberis (Zadoks et al., 2005). In both studies, isolates from feces, the environment, and milk constituted overlapping bacterial populations. It seems reasonable to postulate that this will also be the case for Klebsiella. This hypothesis is supported by the observation that environmental and human clinical isolates of $K$. pneumoniae constitute overlapping populations (Struve and Krogfelt, 2004) and by the ubiquitous and opportunistic nature of Klebsiella (Podschun and Ullmann, 1998; Hogan and Smith, 2003).

The method used for identification of Klebsiella in fecal samples is simple and cheap. It relies on the use of MacConkey agar with ampicillin for sample screening, followed by biochemical testing for confirmation of isolate identity. Genus identity was confirmed for $93 \%$ of Klebsiella-like colonies when isolate selection was aimed at high sensitivity, potentially compromising specificity. As the project progressed, project personnel gained experience in phenotypic recognition of Klebsiella, and specificity of preliminary identification increased (data not shown). The majority of positive samples were identified after a 4-h preincubation, with the exception of samples that were not cooled properly during transport. Across both parts of the study, $13 \%$ of fecal samples were subjected to prolonged incubation, and $30 \%$ of samples subjected to prolonged incubation showed growth of Klebsiella. Thus, the false-negative rate if no prolonged incubation were used would be $4 \%$ of the total number of samples tested.

It could be argued that $K$. pneumoniae is present in feces at low levels if enrichment is needed for detection, and that such low levels may be of limited clinical relevance. However, freshly voided feces would constitute an "inoculant," adding bacteria, organic matter, and nu- 
trients for their growth to sand bedding. Klebsiella levels in used sand in our study and those reported by others (Zdanowicz et al., 2004; Kristula et al., 2005) are below the "generally accepted threshold number of 1,000,000 cfu/g thought to cause mastitis" (Kristula et al., 2005). However, use of a threshold value of $10^{6} \mathrm{cfu} / \mathrm{g}$ as indicator for the risk of mastitis is a matter of habit rather than science (Smith and Hogan, 2006). Factors other than pathogen load also affect teat-end contamination and incidence of mastitis (Zdanowicz et al., 2004; Smith and Hogan, 2006). In our opinion, it is logical and plausible to assume that the occurrence of clinical mastitis in sand-bedded herds can be the result of fecal shedding of $K$. pneumoniae and contamination of bedding with Klebsiella at levels below $10^{6} \mathrm{cfu} / \mathrm{g}$.

\section{CONCLUSIONS}

In conclusion, we used a cheap and simple method to detect presence of Klebsiella in fecal samples of dairy cows, and demonstrated that a large proportion of healthy, adult animals are fecal shedders of $K$. pneumoniae. This finding may explain why use of inorganic bedding reduces the incidence of Klebsiella mastitis relative to organic bedding, but does not result in complete elimination of Klebsiella mastitis from dairy herds, even when the bedding material is free from Klebsiella upon introduction into the stalls. The culture method could be used to further explore the role of fecal shedding in the epidemiology of Klebsiella mastitis; for example, by means of strain typing, and to identify herd- or cowlevel risk factors associated with fecal shedding of Klebsiella, and with the relationship between fecal shedding and clinical mastitis. Even in the absence of wood-based bedding, environmental hygiene is of paramount importance in the control of environmental mastitis, including mastitis caused by Klebsiella.

\section{ACKNOWLEDGMENTS}

We would like to thank participating producers and our colleagues at Quality Milk Production Services for collection of fecal samples and for helpful discussions of culture procedures, Roberta Militello for preparation of graphics, and H. W. Barkema and Y. H. Schukken for critical review of the manuscript.

\section{REFERENCES}

Bagley, S. T. 1985. Habitat association of Klebsiella species. Inf. Control 6:52-58.

Bagley, S. T., and R. J. Seidler. 1977. Significance of fecal coliformpositive Klebsiella. Appl. Environ. Microbiol. 33:1141-1148.

Bagley, S. T., R. J. Seidler, H. W. Talbot Jr., and J. E. Morrow. 1978. Isolation of Klebsielleae from within living wood. Appl. Environ. Microbiol. 36:178-185.
Barkema, H. W., Y. H. Schukken, T. J. G. M. Lam, M. L. Beiboer, H. Wilmink, G. Benedictus, and A. Brand. 1998. Incidence of clinical mastitis in dairy herds grouped in three categories by bulk milk somatic cell counts. J. Dairy Sci. 81:411-419.

Brisse, S., and E. van Duijkeren. 2005. Identification and antimicrobial susceptibility of 100 Klebsiella animal clinical isolates. Vet. Microbiol. 105:307-312.

Degener, J. E., A. C. Smit, M. F. Michel, H. A. Valkenburg, and L. Muller. 1983. Faecal carriage of aerobic gram-negative bacilli and drug resistance of Escherichia coli in different age-groups in Dutch urban communities. J. Med. Microbiol. 16:139-145.

Erskine, R. J., P. C. Bartlett, J. L. VanLente, and C. R. Phipps. 2002a. Efficacy of systemic ceftiofur as a therapy for severe clinical mastitis in dairy cattle. J. Dairy Sci. 85:2571-2575.

Erskine, R. J., J. W. Tyler, M. G. Riddell, Jr., and R. C. Wilson. 1991. Theory, use, and realities of efficacy and food safety of antimicrobial treatment of acute coliform mastitis. J. Am. Vet. Med. Assoc. 198:980-984.

Erskine, R. J., R. D. Walker, C. A. Bolin, P. C. Bartlett, and D. G. White. 2002b. Trends in antibacterial susceptibility of mastitis pathogens during a seven-year period. J. Dairy Sci. 85:1111-1118.

Gröhn, Y. T., D. J. Wilson, R. N. Gonzalez, J. A. Hertl, H. Schulte, G. Bennett, and Y. H. Schukken. 2004. Effect of pathogen-specific clinical mastitis on milk yield in dairy cows. J. Dairy Sci. 87:3358-3374.

Hogan, J., and K. L. Smith. 2003. Coliform mastitis. Vet. Res. 34:507-519.

Hogan, J. S., K. L. Smith, K. H. Hoblet, D. A. Todhunter, P. S. Schoenberger, W. D. Hueston, D. E. Pritchard, G. L. Bowman, L. E. Heider, and B. L. Brockett. 1989. Bacterial counts in bedding materials used on nine commercial dairies. J. Dairy Sci. 72:250-258.

Hogan, J. S., K. L. Smith, D. A. Todhunter, and P. S. Schoenberger. 1990. Bacterial counts associated with recycled newspaper bedding. J. Dairy Sci. 73:1756-1761.

Kristula, M. A., W. Rogers, J. S. Hogan, and M. Sabo. 2005. Comparison of bacteria populations in clean and recycled sand used for bedding in dairy facilities. J. Dairy Sci. 88:4317-4325.

National Mastitis Council. 1999. Laboratory Handbook on Bovine Mastitis. National Mastitis Council, Madison, WI.

Nemeth, J., C. A. Muckle, and C. L. Gyles. 1994. In vitro comparison of bovine mastitis and fecal Escherichia coli isolates. Vet. Microbiol. 40:231-238.

Newman, L. E., and J. J. Kowalski. 1973. Fresh sawdust bedding-a possible source of Klebsiella organisms. Am. J. Vet. Res. 34:979-980.

Podschun, R., and U. Ullmann. 1998. Klebsiella spp. as nosocomial pathogens: Epidemiology, taxonomy, typing methods, and pathogenicity factors. Clin. Microbiol. Rev. 11:589-603.

Roberson, J. R., L. D. Warnick, and G. Moore. 2004. Mild to moderate clinical mastitis: Efficacy of intramammary amoxicillin, frequent milk-out, a combined intramammary amoxicillin, and frequent milk-out treatment versus no treatment. J. Dairy Sci. 87:583-592.

Smith, K. L., and J. S. Hogan. 2006. Bedding counts in manure solids. Page 161-167 in 46th Ann. Mtg. Proc. NMC, Madison, WI.

Smith, K. L., D. A. Todhunter, and P. S. Schoenberger. 1985. Environmental mastitis: Cause, prevalence, prevention. J. Dairy Sci. 68:1531-1553.

Struve, C., and K. A. Krogfelt. 2004. Pathogenic potential of environmental Klebsiella pneumoniae isolates. Environ. Microbiol. 6:584-590.

Todhunter, D. A., K. L. Smith, J. S. Hogan, and P. S. Schoenberger. 1991. Gram-negative bacterial infections of the mammary gland in cows. Am. J. Vet. Res. 52:184-188.

Zadoks, R. N., L. L. Tikofsky, and K. J. Boor. 2005. Ribotyping of Streptococcus uberis from a dairy's environment, bovine feces and milk. Vet. Microbiol. 109:257-265.

Zdanowicz, M., J. A. Shelford, C. B. Tucker, D. M. Weary, and M. A. von Keyserlingk. 2004. Bacterial populations on teat ends of dairy cows housed in free stalls and bedded with either sand or sawdust. J. Dairy Sci. 87:1694-1701. 